\title{
Treatment Persistence Between Long-Acting Injectable Versus Orally Administered Aripiprazole Among Patients with Schizophrenia in a Real-World Clinical Setting in Japan
}

\author{
Nakao Iwata - Ataru Inagaki · Hiromi Sano - Kazunari Niidome • \\ Yoshitsugu Kojima · Sakiko Yamada
}

Received: February 27, 2020 / Published online: June 4, 2020

(C) The Author(s) 2020

\begin{abstract}
Introduction: Persistence with antipsychotic treatment is critical in managing patients with schizophrenia. To evaluate whether aripiprazole long-acting injection (aripiprazole oncemonthly, AOM) can contribute to longer treatment persistence compared with daily orally administered aripiprazole (OA) in real-world clinical settings in Japan, treatment persistence in patients with schizophrenia was compared
\end{abstract}

Digital Features To view digital features for this article go to https://doi.org/10.6084/m9.figshare.12370313.

Electronic supplementary material The online version of this article (https://doi.org/10.1007/s12325020-01396-w) contains supplementary material, which is available to authorized users.

N. Iwata

Department of Psychiatry, Fujita Health University, Aichi, Japan

A. Inagaki

Department of Education, College of Education, Psychology and Human Studies, Aoyama Gakuin University, Tokyo, Japan

H. Sano $(\square)$

Medical Affairs, Otsuka Pharmaceutical Co., Ltd., Osaka, Japan

e-mail: sanoh@otsuka.jp

K. Niidome $\cdot$ Y. Kojima $\cdot$ S. Yamada

Medical Affairs, Otsuka Pharmaceutical Co., Ltd.,

Tokyo, Japan between patients treated with AOM and those with OA, using a claims database compiled by JMDC Inc., Tokyo, Japan.

Methods: Data of patients with schizophrenia who newly initiated AOM or OA treatment between May 2015 and November 2017 were analyzed. The Cox proportional hazard model was used to estimate the hazard ratio (HR) for treatment discontinuation of $\mathrm{AOM}$ vs. OA treatment, adjusted for age, sex, chlorpromazine-equivalent dose of antipsychotics, and the number of psychiatric hospitalizations.

Results: The analysis included 198 patients in the AOM group and 1240 patients in the OA group (mean age $38.4 \pm 11.9$ years and $39.3 \pm 12.4$ years, respectively). The AOM group was significantly less likely to discontinue treatment than the OA group (adjusted HR 0.54, 95\% confidence interval [CI] 0.43-0.68). When using the tolerable patients extracted from the OA group (i.e., patients with at least two OA prescriptions; $n=983$ ) vs. the whole AOM group, AOM users were again significantly less likely to discontinue treatment (adjusted HR 0.67, 95\% CI 0.53-0.86).

Conclusion: AOM was associated with longer treatment persistence than OA in the antipsychotic treatment of patients with schizophrenia in real-world clinical settings in Japan, suggesting that the use of AOM may contribute to longer antipsychotic treatment. 
Keywords: Antipsychotic; Aripiprazole oncemonthly; Continuation rate; Japan; Longacting injectable; Schizophrenia

\section{Key Summary Points}

Why carry out this study?

Poor adherence leads to worse prognosis, longer time to remission, and increased risks of relapse and hospitalization in patients with schizophrenia

Although the use of an antipsychotic longacting injection (LAI) is recommended as a way of improving medication adherence, LAIs remain underused in Japan

This study evaluated whether patients with schizophrenia receiving the LAI formulation of aripiprazole (aripiprazole once-monthly, AOM) indeed have better treatment persistence than those receiving orally administered aripiprazole $(\mathrm{OA})$ in real-world clinical settings in Japan

\section{What was learned from the study?}

Patients treated with AOM had significantly longer treatment persistence than those treated with OA

The use of AOM may contribute to prolonging treatment persistence among patients with schizophrenia, for whom such persistence is extremely important

\section{INTRODUCTION}

Antipsychotic treatment is the standard treatment in patients with schizophrenia, which aims not only to improve acute symptoms but also to maintain long-term stabilization of symptoms and prevent relapses. However, nonadherence is quite common among patients with schizophrenia [1], with the prevalence of nonadherence being $19.2-73 \%$ in 21 studies [2]. As poor adherence leads to worse prognosis, longer time to remission, and increased risks of relapse, hospitalization, and suicide $[1,3,4]$, improvement of adherence to antipsychotic treatment or achievement of treatment persistence is important for the treatment of patients with schizophrenia.

Practice guidelines recommend the use of long-acting injectable (LAI) antipsychotics as a way of improving medication adherence $[5,6]$. LAIs, which are commonly administered on a biweekly or monthly basis, reduce the burden of daily oral antipsychotic use and make early detection of nonadherence easier [7]. LAIs have at least similar treatment outcomes (e.g., relapse prevention) to oral antipsychotics [8,9], with some studies reporting superiority of LAIs over oral treatments [10-12]. In addition, patients who initiated LAIs were reportedly more adherent and had longer time to treatment discontinuation than those receiving oral antipsychotics in several non-randomized studies [7, 13-17]. Although LAIs used to be reserved for patients with frequent relapses or poor adherence [5], the early use of LAIs is increasingly being advocated [10, 18, 19] and recommended for consideration as a first-line treatment for any patient for whom long-term antipsychotic treatment is needed [20]. Nevertheless, LAIs have been underused as a result of lack of knowledge and negative attitudes about LAIs among clinicians and patients [10, 18].

Aripiprazole is a second-generation antipsychotic with a high affinity partial agonism of dopamine $\mathrm{D}_{2}$ receptors and serotonin $5-\mathrm{HT}_{1 \mathrm{~A}}$ receptors and an antagonism of $5-\mathrm{HT}_{2 \mathrm{~A}}$ receptors [21], with efficacy in the acute and longterm treatment, and low incidence of side effects such as weight gain, hyperprolactinemia, and metabolic adverse events [22]. A oncemonthly LAI formulation of aripiprazole (aripiprazole once-monthly, AOM) was developed to improve patients' adherence, which was first approved in the USA in 2013 [23] and later marketed in Japan in May 2015 [24]. AOM was shown to have comparable efficacy and tolerability to orally administered aripiprazole (OA) in an Asian randomized study [8]; however, there was no significant difference in treatment 
persistence between the two formulations. Outside Asia, a few studies previously suggested the advantage of AOM for the longer treatment persistence over oral antipsychotics in realworld clinical settings $[15,16]$, but these were not direct comparisons with OA. If real-world evidence suggests such an advantage of AOM over OA in long-term treatment persistence, it would add to clinicians' knowledge and may change their attitudes toward the use of the formulation, which could potentially encourage its use in clinical practice. Therefore, in this study, we compared the treatment persistence between patients with schizophrenia who initiated AOM and those who initiated OA in realworld clinical settings in Japan, using a largescale claims database.

\section{METHODS}

\section{Study Design and Data Source}

This was a retrospective, observational cohort study in patients with schizophrenia who newly initiated treatment with $\mathrm{AOM}$ or OA, using a claims database compiled by JMDC Inc., Tokyo, Japan.

The JMDC database is based on insured persons' claims (in-patient, outpatient, and pharmacy) received from medical institutions, and it includes data of about four million persons as of May 2019. The individuals included in the database are company employees and their dependents insured by health insurance societies. Contained data, which were securely anonymized, included patient demographics (age, sex), diagnoses, prescriptions, medical procedures, costs, and medical institutions (e.g., bed size). A unique identifier is assigned to each person to link their claims records across institutions, which enables one to track their movement and treatment across institutions as long as they are covered by health insurance societies. The JMDC database has been used in various areas of medical research in Japan, including studies in patients with schizophrenia $[25,26]$. The present study used data from May 1, 2014 to November 30, 2018 (entire data period). This study was approved by the ethics committee of the Research Institute of Health Data Science (RI2019001) and was conducted according to the principles of the Declaration of Helsinki.

\section{Patient Selection}

Patients aged 18 years or more with a confirmed diagnosis of schizophrenia (International Statistical Classification of Diseases and Related Health Problems 10th Revision [ICD-10] code F20) who had a prescription record of AOM or OA (index drug) between May 1, 2015 and November 30, 2017, with no prescription records of the index drug in the 365 days prior to index date were identified within the database. This time frame, beginning in May of 2015, was chosen to compare the two treatments after AOM became available in Japan. Index date was the first date of index drug prescription with a diagnosis record of schizophrenia (Fig. 1). To target patients who had been receiving antipsychotic treatment continuously, patients were required to have both a diagnosis record for schizophrenia and a prescription record for antipsychotics on admission to hospital or at two or more outpatient visits in the 365-day preindex period. In this study, both the diagnosis and the prescription records were considered conditions for patient identification. This approach was adopted to reduce possible misclassification in a previous claims database study of schizophrenia [27].

The following patients were excluded: (1) patients with less than a $150 \mathrm{mg} /$ day chlorpromazine (CP)-equivalent dose of antipsychotics and also with a prescription record of antidepressants in the 365-day pre-index period; and (2) patients with a diagnosis record of dementia (ICD-10 codes: F00-03, F05.1, G30, G31.1), autism (F84), or intellectual disability (F70-79) in the month of index date or during the 12-month pre-index period. Patients with a prescription record of AOM any time in the entire data period were also excluded from the OA group. The concomitant use of multiple antipsychotics, irrespective of the formulation, was not an exclusion criterion in this study, excepting the use of AOM in the OA group. 


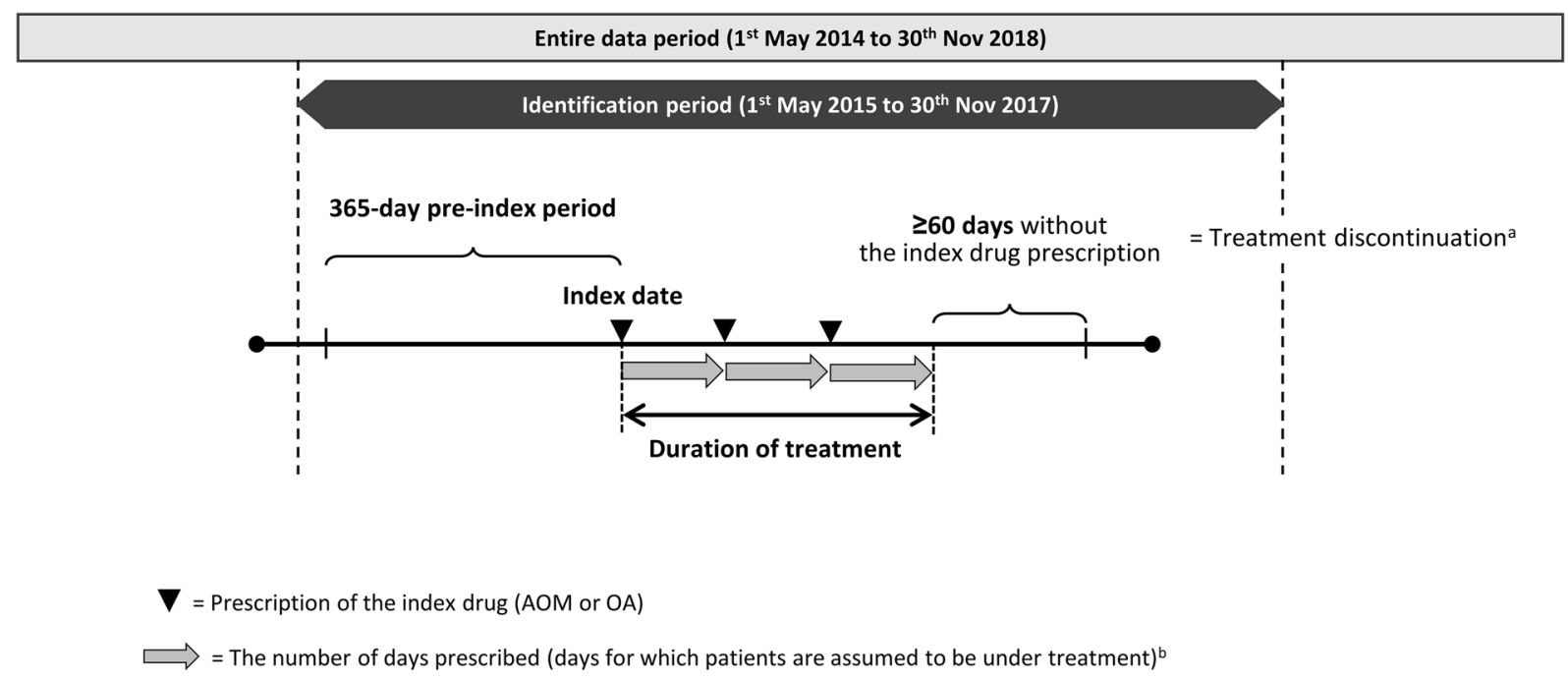

Fig. 1 Study time frame. Index date is the earliest date of prescription of the index drug ( $\mathrm{AOM}$ or $\mathrm{OA}$ ) for the treatment of schizophrenia within the identification period. ${ }^{a}$ In sensitivity analyses, at least 30 days and at least

\section{Outcomes}

The primary outcome was the duration of index drug treatment. For OA, patients were assumed to have taken aripiprazole for the number of days prescribed recorded on claims. For AOM, patients were assumed to have been on medication for 28 days per prescription. Discontinuation was defined as at least 60 days absence of subsequent prescriptions following the last day of the days prescribed. Treatment persistence was defined as the number of days from index date to the last day of the days prescribed by the latest prescription before discontinuation (Fig. 1). When the 60-day gap could not be confirmed because of the lack of data (e.g., end of the data period or withdrawn from the database), treatment was censored at the last day of data observed, and treatment persistence was defined to end on that day. In sensitivity analyses, the at least 30-day and at least 90-day gaps were used as the definition of discontinuation.

Background characteristics of patients, including the following variables, were measured using data for the 365-day pre-index period, unless otherwise described: age at index
90 days were used as definitions of discontinuation. ${ }^{b}$ For AOM, 28 days per prescription. For OA, the number of days prescribed recorded on claims. AOM aripiprazole once-monthly, OA orally administered aripiprazole

date, sex, comorbidity score, psychiatric comorbidities in index month, concomitant psychotropics (antidepressants, mood stabilizers, anxiolytics, hypnotics) in index month, CPequivalent dose of antipsychotics (mg/day), proportion of days covered (PDC) for antipsychotics, and hospitalizations. A comorbidity score was calculated on the basis of the Charlson Comorbidity Index [28] but excluded AIDS/ HIV because of the unavailability of data. PDC was calculated as the total number of days prescribed for antipsychotics during the treatment period divided by the number of days in the period.

\section{Analysis Sets}

The analysis sets in this study were defined as follows:

1. Overall analysis set (AOM, $n=198$; OA, $n=1240$ ), consisting of all patients who met all inclusion criteria without meeting any exclusion criteria.

2. Tolerable OA subset $(n=983)$, consisting of patients with at least two prescription records of $\mathrm{OA}$ among the overall analysis set of the OA group. 
3. No-antidepressant subset (AOM, $n=152$; OA, $n=837$ ), consisting of patients without prescriptions for antidepressants in the 365-day pre-index period.

4. Maintenance dose subset (AOM, $n=193$; OA, $n=917$ ), consisting of patients with at least a $150 \mathrm{mg} /$ day CP-equivalent dose of antipsychotics in the 365-day pre-index period. A dose of $150 \mathrm{mg} /$ day is the minimum maintenance dose of OA for schizophrenia in Japan.

\section{Statistical Analysis}

Patient characteristics were descriptively summarized for each group. The Cox proportional hazard model was used to compare the treatment persistence between $\mathrm{AOM}$ and $\mathrm{OA}$, and hazard ratios (HRs) for treatment discontinuation and 95\% confidence intervals (CIs) were computed. The multivariable model included the following background characteristics of patients as covariates: age (less than 40 , at least 40 years), sex, CP-equivalent dose of antipsychotics (less than 600, at least $600 \mathrm{mg} /$ day), and the number of psychiatric hospitalizations $(0$, at least 1). In addition, adjusted Cox regression survival curves were generated to depict the treatment continuation rate in each treatment group.

The AOM group was assumed to include tolerable patients because tolerability of aripiprazole must be established with OA prior to the initiation of AOM [24], whereas the tolerability was unknown among patients initiating $\mathrm{OA}$. To reduce potential bias, we also conducted the same analysis as above using the tolerable OA subset and compared their treatment persistence against the overall AOM group. In this subset, the treatment persistence was defined to start at the second prescription date of OA, not at index date.

In addition, we performed two subgroup analyses to further examine the treatment persistence between AOM and OA by eliminating potential confounding effects from the unintended use of the index drug. First, the no-antidepressant subset was analyzed to eliminate the potential use of aripiprazole as an augmentation therapy for depression. Second, the maintenance dose subset was analyzed to eliminate patients on a dose below the maintenance dose of antipsychotics for schizophrenia treatment. This suggests a potential use of aripiprazole for depression or other treatment purposes.

The level of significance was set at $p<0.05$ (two-sided). All statistical analyses were conducted using SAS 9.4 (SAS Institute Inc., Cary, NC). Statistical analyses were independently conducted by JMDC Inc. (Tokyo, Japan).

\section{RESULTS}

\section{Patient Characteristics}

The present study included 198 patients for the AOM group and 1240 patients for the OA group (= overall analysis set; Fig. 2). The background characteristics of patients are summarized in Table 1 . The age and sex distribution was similar between the AOM and OA groups (mean age $38.4 \pm 11.9$ years vs. $39.3 \pm 12.4$ years, respectively; proportion of women, $63.1 \%$ vs. $59.4 \%$, respectively). In the index month, OA users had comorbid depressive disorders more commonly than AOM users (41.1\% vs. $27.8 \%$, respectively) and used more antidepressants $(25.0 \%$ vs. $12.6 \%$, respectively). The mean CP-equivalent dose of antipsychotics during the 365-day preindex period was $626.5 \pm 481.6 \mathrm{mg} /$ day in the AOM group and $346.3 \pm 369.0 \mathrm{mg} /$ day in the OA group. During the pre-index period, patients in the AOM group had experienced psychiatric hospitalizations more than those in the OA group ( $41.9 \%$ vs. $25.0 \%$, respectively). The proportions of patients who were hospitalized at index date were similar between the AOM and OA groups (20.2\% vs. $17.2 \%$, respectively).

\section{Treatment Persistence}

The analyses using the Cox proportional hazard models revealed that patients treated with AOM were significantly less likely to discontinue treatment (adjusted HR 0.54, 95\% CI 0.43-0.68; Table 2 and Fig. 3a). When the tolerable OA 


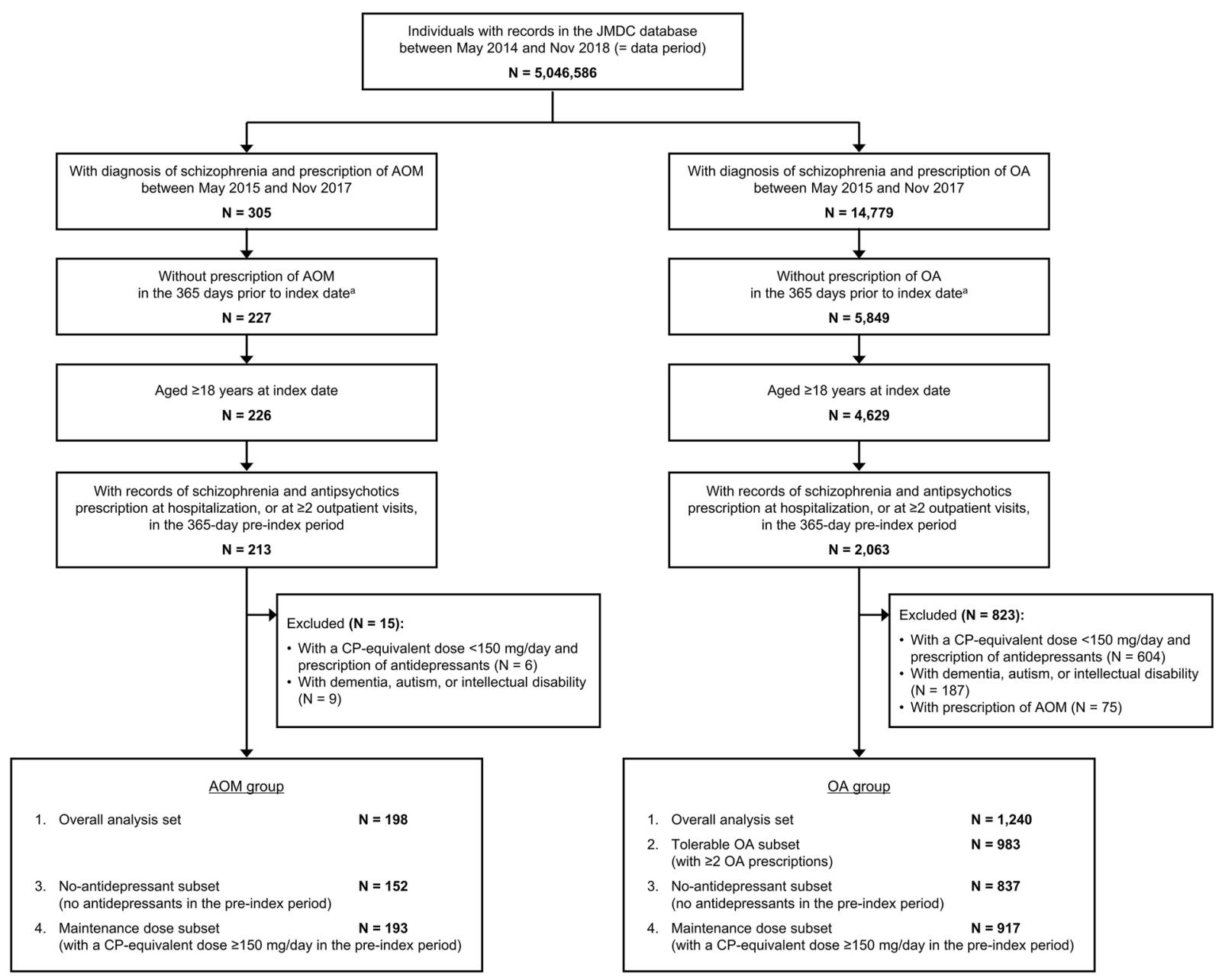

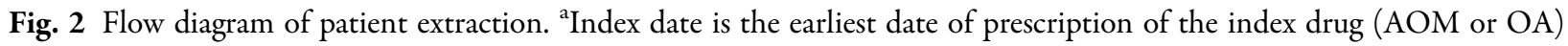
with diagnosis of schizophrenia. AOM aripiprazole once-monthly, CP chlorpromazine, OA orally administered aripiprazole

subset was used, AOM users were again significantly less likely to discontinue treatment (adjusted HR 0.67, 95\% CI 0.53-0.86; Fig. 3b). In our sensitivity analyses using the overall analysis set, the alternative definitions of at least 30-day and at least 90-day gaps as treatment discontinuation both yielded similar results showing significantly lower risk for treatment discontinuation in the AOM group (Supplementary Table S1).

The subgroup analysis in the no-antidepressant subset demonstrated that patients treated with AOM were still significantly less likely to discontinue treatment than those treated with OA (adjusted HR 0.53, 95\% CI 0.40-0.69). Another subgroup analysis using the maintenance dose subset also revealed that AOM users were significantly less likely to discontinue treatment than OA users (adjusted HR $0.54,95 \%$ CI 0.43-0.69).

\section{DISCUSSION}

This is a unique study comparing the treatment persistence in patients with schizophrenia between AOM and OA in real-world clinical settings in Japan. A meta-analysis of randomized controlled trials (RCTs) on AOM previously reported the lower risk for all-cause discontinuation in AOM vs. OA (relative risk [RR] 0.78, 95\% CI 0.64-0.95) [29]. However, because RCTs, which are conducted under strict conditions, 
Table 1 Background characteristics of patients in the AOM and OA groups (overall analysis set)

AOM

$(n=198)$

$38.4 \pm 11.9$

$125(63.1)$

$55(27.8)$

$35(17.7)$

$1(0.5)$

$25(12.6)$

$43(21.7)$

57 (28.8)

$107(54.0)$

$2.1 \pm 5.4$

35 (17.7)

$40(20.2)$

$0.5 \pm 1.0$

$626.5 \pm 481.6$

$5(2.5)$

33 (16.7)

77 (38.9)

83 (41.9)

$0.79 \pm 0.27$

133 (67.2)

$0.5 \pm 0.7$

83 (41.9)
OA

$(n=1240)$

$39.3 \pm 12.4$

$736(59.4)$

$510(41.1)$

331 (26.7)

$26(2.1)$

$310(25.0)$

316 (25.5)

440 (35.5)

$710(57.3)$

$9.6 \pm 15.1$

728 (58.7)

$213(17.2)$

$0.5 \pm 1.1$

$346.3 \pm 369.0$

$323(26.0)$

437 (35.2)

$282(22.7)$

$198(16.0)$

PDC for antipsychotics

$$
\begin{aligned}
& \text { Mean } \pm \text { SD } \\
& \geq 0.8, n(\%)
\end{aligned}
$$

$0.79 \pm 0.26$

830 (66.9)

Number of psychiatric hospitalizations

$$
\begin{aligned}
& \text { Mean } \pm \text { SD } \\
& \geq 1, n(\%)
\end{aligned}
$$

$0.3 \pm 0.5$ $310(25.0)$

$83(41.9)$


Table 1 continued

\begin{tabular}{lll}
\hline & $\begin{array}{l}\text { AOM } \\
(\boldsymbol{n}=\mathbf{1 9 8})\end{array}$ & $\begin{array}{l}\text { OA } \\
(\boldsymbol{n}=\mathbf{1 2 4 0})\end{array}$ \\
\hline Total length of hospital-stay, days & $43.2 \pm 78.3$ & $20.7 \pm 53.4$ \\
Time intervals between antipsychotic prescriptions $^{\mathrm{b}}$, days & $31.6 \pm 34.6$ & $28.9 \pm 27.4$ \\
Use of other LAI antipsychotics, $n(\%)^{\text {Time from the oldest OA prescription to index date, days }}$ & $33(16.7)$ & $38(3.1)$ \\
Dose of OA at most recent prescription, mg/day & $204.3 \pm 147.1$ & - \\
\hline
\end{tabular}

Data are expressed as mean \pm SD or $n(\%)$

$A D H D$ attention-deficit hyperactivity disorder, $A O M$ aripiprazole once-monthly, $C P$ chlorpromazine, $D Z P$ diazepam, $L A I$ long-acting injectable, $O A$ orally administered aripiprazole, $P D C$ proportion of days covered, $S D$ standard deviation

a Comorbidity score was calculated on the basis of the Charlson Comorbidity Index but excluded AIDS/HIV because of the unavailability of data

b Time intervals between outpatient prescriptions of antipsychotics during the 365-day pre-index period were calculated

Table 2 Hazard ratios for treatment discontinuation (overall analysis set)

\begin{tabular}{|c|c|c|c|c|}
\hline & \multicolumn{2}{|c|}{ Unadjusted model } & \multicolumn{2}{|l|}{ Adjusted model $^{a}$} \\
\hline & $\overline{\mathrm{HR}(95 \% \mathrm{CI})}$ & $p$ value & $\overline{\mathrm{HR}(95 \% \mathrm{CI})}$ & $p$ value \\
\hline AOM vs. OA & $0.50(0.40-0.63)$ & $<0.0001$ & $0.54(0.43-0.68)$ & $<0.0001$ \\
\hline
\end{tabular}

would not reflect the reality of daily clinical practice, real-world evidence should confirm such a potential advantage of AOM over OA in treatment persistence. In this claims data study, we found that patients who initiated AOM were, indeed, less likely to discontinue treatment compared with those who initiated OA in routine clinical practice in Japan (adjusted HR $0.54,95 \%$ CI $0.43-0.68$ ). Our findings are consistent with trends observed in other claims data studies showing that AOM users in the USA are less likely to discontinue treatment (HR 0.69, 95\% CI 0.60-0.79) [15] and have significantly longer time to discontinuation than oral antipsychotic users [16].

Although our results showed the advantage of $\mathrm{AOM}$ in treatment continuation, there were some biases to be considered when interpreting the results of this study. For example, the tolerability of aripiprazole had not been confirmed prior to treatment initiation among the OA group, whereas it had most probably been confirmed before initiating AOM. Because of this, early dropouts due to intolerability must have contributed to the lower continuation rates among the OA group. However, our analysis using tolerable patients extracted from the OA group still demonstrated significantly lower risk for treatment discontinuation in the AOM treatment (adjusted HR 0.67, 95\% CI $0.53-0.86$ ). The presence of at least two prescriptions does not necessarily confirm the tolerability; however, this condition could at least exclude some OA-administered patients who discontinued treatment early, e.g., after initial administration. Thus, we consider that the results of this analysis corroborate our finding.

$\mathrm{OA}$ is also indicated for major depressive disorders with inadequate response to antidepressant therapy [30], and its efficacy as an augmentation therapy has been well documented [31, 32]. Considering the high 
(a) AOM vs. OA groups (overall analysis set)

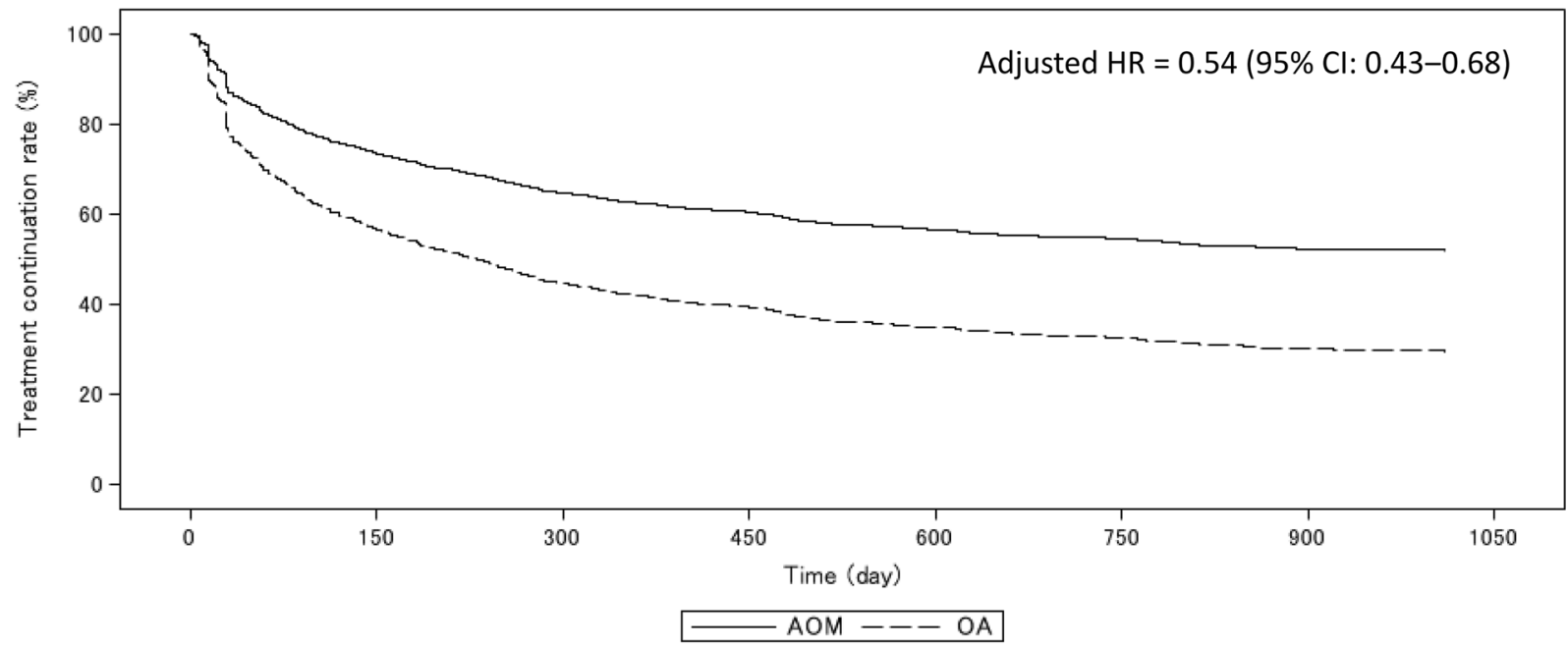

(b) AOM group vs. tolerable OA subset

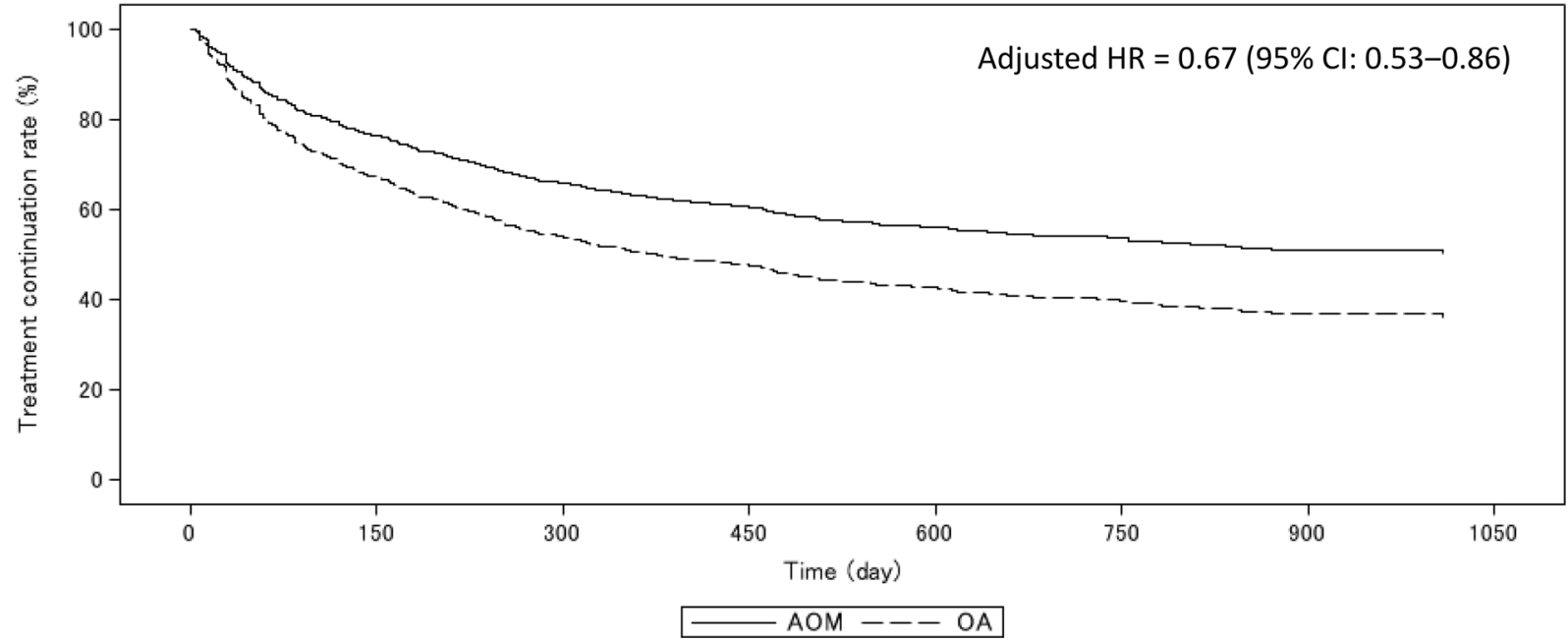

Fig. 3 Adjusted Cox regression survival curves for treatment discontinuation in each treatment group. a AOM and OA groups were compared. b The tolerable OA subset was compared against the AOM group. Adjusted for age,

prevalence of comorbid depressive disorders in the OA group (41.1\%), the possible use of OA as augmentation therapy for depressive disorders may have been responsible, in part, for the shorter treatment persistence in the OA group. To address this point, we conducted a subgroup analysis using patients without prescriptions of antidepressants, for whom OA must have been administered exclusively for the treatment of sex, the CP-equivalent dose of antipsychotics (mg/day), and the number of psychiatric hospitalizations. AOM aripiprazole once-monthly, CP chlorpromazine, OA orally administered aripiprazole

schizophrenia. However, even among these patients, we found that AOM users still had a significantly lower risk for treatment discontinuation than OA users (adjusted HR 0.53, 95\% CI 0.40-0.69). Similarly, patients with only less than the maintenance dose of antipsychotics (26.0\% in the OA group vs. $2.5 \%$ in the AOM group), who had probably received aripiprazole for other treatment purposes, might also have 
influenced the results of this study. However, again, the analysis after excluding these patients also showed that AOM users were still significantly less likely to discontinue treatment than OA users (adjusted HR 0.54, 95\% CI 0.43-0.69). Altogether, we reasoned that these results supported the advantage of AOM over OA in treatment continuation even when considering these potential biases.

In Japan, LAIs remain underused [27] probably because of negative attitudes toward the use of LAIs among Japanese psychiatrists, such as little experience with LAIs, concerns for switching, fear of pain, and high drug costs [33]. However, a recent Japanese study reported that patients treated with LAIs had favorable attitudes toward LAIs regarding side effects, relapse prevention, efficacy, pain, and costs [34]. Considering that LAI antipsychotics offer a simpler mode of administration than oral antipsychotics, with the latter having more barriers to treatment adherence (e.g., hospital visit for a prescription form, pharmacy visit for drug purchase, daily drug intake), some patients may favor LAI formulations for simplicity. Thus, the use of LAIs should be encouraged among both psychiatrists and patients, beyond the cases of poor adherence and high risks for recurrence $[18,35,36]$. We expect that the results of this study add to the knowledge on AOM among Japanese psychiatrists, thereby contributing to its broader use in clinical settings.

The focus of this study was on the difference in treatment continuation between two formulations of aripiprazole; however, the observed trend favoring an LAI formulation over its oral counterpart was consistent with a previous report that included haloperidol, perphenazine, and risperidone [37]. This also paralleled the trend of better adherence with risperidone LAI compared with orally administered risperidone [12]. Our results would also add to the benefits of LAIs compared with oral antipsychotics in terms of a longer treatment duration, previously reported for paliperidone, risperidone, and haloperidol $[7,14,15]$. Now that the advantages of LAIs over oral antipsychotics in treatment persistence seem to be evident, the next research topic may be a comparison among LAIs. Since there are several LAI antipsychotics available in Japan, it would be worth investigating the benefits of an AOM relative to other LAI antipsychotics in a future study.

There were several limitations in this study. First, because the JMDC database only includes insured individuals covered by health insurance societies, patients with different socioeconomic background, who may be covered by other health insurance policies, and those aged 65 years and older were quite limited in our study population. Therefore, the generalizability of our results to the overall patients with schizophrenia in Japan may be limited. Second, as data were originally recorded for the purpose of claims rather than research, it is possible that recorded diagnosis and treatment may be inaccurate, influencing the results of this study. Third, it has been suggested that an AOM is a more direct indicator of medication adherence, as the prescription record most probably indicates actual injection of the AOM. In contrast, the process of drug administration for the OA group is complicated [38]. Given the potential failures of drug intake in the OA group, the between-group gap in continuation rates may have been greater. Fourth, there were some differences in patients' background characteristics between the AOM and OA groups, probably reflecting the selection bias in real-world clinical settings, which suggest the potential of confounding. Our subgroup analyses showed that the results were consistent even after excluding patients with antidepressants and the extremely low CP-equivalent dose of antipsychotics, demonstrating that the impact of differences in these two variables was not substantial. The same trend was also observed when other additional, potentially relevant, patient characteristics were considered in our exploratory analysis (see Supplementary Table S2). However, there might have been other factors influencing the results of this study, such as patients' severity or psychiatrists' attitudes toward the use of LAIs. We could not assess the effects of these factors because of the absence of such data within the database; these are worth investigating in future research to confirm the result of this study. Finally, and most importantly, we were unable to evaluate the reasons for treatment discontinuation or 
switching because of the absence of data. A future study should clarify such reasons or causes, which would help develop strategies to improve patients' adherence to treatment.

\section{CONCLUSIONS}

This large-scale claims data study showed that patients with schizophrenia receiving AOM had significantly longer treatment persistence with an adjusted HR for treatment discontinuation of 0.54 , compared with those receiving OA in realworld clinical settings in Japan. Although there were some important biases and study limitations to be considered, additional analyses, including sensitivity and subgroup analyses, yielded consistent results, supporting the advantage of AOM over OA in treatment continuation in routine practice. The use of AOM may contribute to prolonging treatment persistence among patients with schizophrenia, for whom such persistence is extremely important.

\section{ACKNOWLEDGEMENTS}

Funding. This work was supported by Otsuka Pharmaceutical Co., Ltd., and the journal's Rapid Service and Open Access Fees were also funded by Otsuka Pharmaceutical Co., Ltd.

Authorship. All named authors meet the International Committee of Medical Journal Editors (ICMJE) criteria for authorship for this article, take responsibility for the integrity of the work as a whole, and have given their approval for this version to be published.

Medical Writing, Editorial, and Other Assistance. We thank Dr. Yasuyuki Okumura (Initiative for Clinical Epidemiological Research) for his advice in statistical analysis planning. We thank Ms. Chie Ito (JMDC, Inc.) for her assistance in the development of statistical analysis plan, Dr. Hidetsugu Tsubouchi (Otsuka Pharmaceutical Co., Ltd.) for editorial support, and Mr. Noriyuki Mamiya (Otsuka Pharmaceutical Co., Ltd.) for his support in study planning, statistical analysis planning, and editing of the manuscript. Medical writing assistance was provided by Clinical Study Support, Inc., and support for this assistance was funded by Otsuka Pharmaceutical Co., Ltd.

Disclosures. Nakao Iwata has received research support or speakers' honoraria from Daiichi-Sankyo Co., Ltd.; Eisai Co., Ltd.; Eli Lilly Japan K.K.; Otsuka pharmaceutical Co., Ltd.; Pfizer Japan Inc.; Sumitomo Dainippon Pharma Co., Ltd.; and Janssen Pharmaceutical K.K. Ataru Inagaki has received manuscript or speaker's fees from Janssen Pharmaceutical K.K.; Meiji Seika Pharma Co., Ltd.; Otsuka Pharmaceutical Co., Ltd.; Yoshitomiyakuhin Corporation; and Takeda Pharmaceutical Co., Ltd. Hiromi Sano, Kazunari Niidome, Yoshitsugu Kojima, and Sakiko Yamada are employees of Otsuka Pharmaceutical Co., Ltd.

Compliance with Ethics Guidelines. This study was approved by the ethics committee of the Research Institute of Health Data Science (reference no. RI2019001) and was conducted according to the principles of the Declaration of Helsinki. Informed consent was not required for this retrospective database study because this study exclusively used de-identified claims data without involving any human participants.

Data Availability. The dataset generated during and/or analyzed during the current study are not publicly available because it was purchased from a commercial provider, but is available from the corresponding author on reasonable request.

Open Access. This article is licensed under a Creative Commons Attribution-NonCommercial 4.0 International License, which permits any non-commercial use, sharing, adaptation, distribution and reproduction in any medium or format, as long as you give appropriate credit to the original author(s) and the source, provide a link to the Creative Commons licence, and indicate if changes were made. The images or other third party material in this article are included in the article's Creative Commons licence, unless indicated otherwise in a credit 
line to the material. If material is not included in the article's Creative Commons licence and your intended use is not permitted by statutory regulation or exceeds the permitted use, you will need to obtain permission directly from the copyright holder. To view a copy of this licence, visit http://creativecommons.org/licenses/by$\mathrm{nc} / 4.0 /$.

\section{REFERENCES}

1. Acosta FJ, Hernández JL, Pereira J, Herrera J, Rodríguez CJ. Medication adherence in schizophrenia. World J Psychiatry. 2012;2:74-82.

2. Svestka J, Bitter I. Nonadherence to antipsychotic treatment in patients with schizophrenic disorders. Neuro Endocrinol Lett. 2007;28:95-116.

3. Higashi K, Medic G, Littlewood KJ, Diez T, Granström $\mathrm{O}$, De Hert M. Medication adherence in schizophrenia: factors influencing adherence and consequences of nonadherence, a systematic literature review. Ther Adv Psychopharmacol. 2013;3: 200-18.

4. Morken G, Widen JH, Grawe RW. Non-adherence to antipsychotic medication, relapse and rehospitalisation in recent-onset schizophrenia. BMC Psychiatry. 2008;8:32.

5. Lehman AF, Lieberman JA, Dixon LB, et al. Practice guideline for the treatment of patients with schizophrenia. Am J Psychiatry. 2004;161:1-56.

6. Velligan DI, Weiden PJ, Sajatovic M, et al. Strategies for addressing adherence problems in patients with serious and persistent mental illness: recommendations from the expert consensus guidelines. J Psychiatr Pract. 2010;16:306-24.

7. Marcus SC, Zummo J, Pettit AR, Stoddard J, Doshi JA. Antipsychotic adherence and rehospitalization in schizophrenia patients receiving oral versus long-acting injectable antipsychotics following hospital discharge. J Manag Care Spec Pharm. 2015;21:754-68.

8. Ishigooka J, Nakamura J, Fujii Y, et al. Efficacy and safety of aripiprazole once-monthly in Asian patients with schizophrenia: a multicenter, randomized, double-blind, non-inferiority study versus oral aripiprazole. Schizophr Res. 2015;161:421-8.

9. Kishimoto T, Robenzadeh A, Leucht C, et al. Longacting injectable vs oral antipsychotics for relapse prevention in schizophrenia: a meta-analysis of randomized trials. Schizophr Bull. 2014;40: 192-213.

10. Kaplan G, Casoy J, Zummo J. Impact of long-acting injectable antipsychotics on medication adherence and clinical, functional, and economic outcomes of schizophrenia. Patient Prefer Adherence. 2013;7: 1171-80.

11. Kishimoto T, Hagi K, Nitta M, et al. Effectiveness of long-acting injectable vs oral antipsychotics in patients with schizophrenia: a meta-analysis of prospective and retrospective cohort studies. Schizophr Bull. 2018;44:603-19.

12. Subotnik KL, Casaus LR, Ventura J, et al. Long-acting injectable risperidone for relapse prevention and control of breakthrough symptoms after a recent first episode of schizophrenia: a randomized clinical trial. JAMA Psychiatry. 2015;72:822-9.

13. Greene M, Yan T, Chang E, Hartry A, Touya M, Broder MS. Medication adherence and discontinuation of long-acting injectable versus oral antipsychotics in patients with schizophrenia or bipolar disorder. J Med Econ. 2018;21:127-34.

14. Pilon D, Tandon N, Lafeuille M-H, et al. Treatment patterns, health care resource utilization, and spending in Medicaid beneficiaries initiating second-generation long-acting injectable agents versus oral atypical antipsychotics. Clin Ther. 2017;39: 1972-1985 e2.

15. Song X, El Khoury AC, Brouillette M, Smith D, Joshi $\mathrm{K}$. Treatment discontinuation of long-acting injectables or oral atypical antipsychotics among Medicaid recipients with schizophrenia. J Med Econ. 2019;22:1105-12.

16. Yan T, Greene M, Chang E, Hartry A, Touya M, Broder MS. Medication adherence and discontinuation of aripiprazole once-monthly $400 \mathrm{mg}$ (AOM $400)$ versus oral antipsychotics in patients with schizophrenia or bipolar I disorder: a real-world study using US claims data. Adv Ther. 2018;35: 1612-25.

17. Zhu B, Ascher-Svanum H, Shi L, Faries D, Montgomery W, Marder SR. Time to discontinuation of depot and oral first-generation antipsychotics in the usual care of schizophrenia. Psychiatr Serv. 2008;59:315-7.

18. Correll CU, Citrome L, Haddad PM, et al. The use of long-acting injectable antipsychotics in schizophrenia: evaluating the evidence. J Clin Psychiatry. 2016;77:1-24.

19. Stevens GL, Dawson G, Zummo J. Clinical benefits and impact of early use of long-acting 
injectable antipsychotics for schizophrenia. Early Interv Psychiatry. 2016;10:365-77.

20. Llorca PM, Abbar M, Courtet P, Guillaume S, Lancrenon S, Samalin L. Guidelines for the use and management of long-acting injectable antipsychotics in serious mental illness. BMC Psychiatry. 2013;13:340.

21. Burris KD, Molski TF, Xu C, et al. Aripiprazole, a novel antipsychotic, is a high-affinity partial agonist at human dopamine D2 receptors. J Pharmacol Exp Ther. 2002;302:381-9.

22. Hirose T, Mamiya N, Yamada S, Taguchi M, Kameya $\mathrm{T}$, Kikuchi T. The antipsychotic drug aripiprazole (ABILIFY®). Folia Pharmacol Jpn. 2006;128:331-45 (Japanese).

23. ABILIFY MAINTENA: Highlights of prescribing information. https://www.accessdata.fda.gov/ drugsatfda_docs/label/2013/202971s000lbl.pdf. Accessed 18 Dec 2019.

24. ABILIFY ${ }^{\circledR}$ prolonged release aqueous suspension for IM injection [package insert]. Japanese. https:// www.otsuka-elibrary.jp/di/prod/product/file/abj/ abjbnotk.pdf. Accessed 18 Dec 2019.

25. Ye W, Nakahara N, Takahashi M, Ascher-Svanum H. Characteristics of outpatients initiated on olanzapine versus risperidone in the treatment of schizophrenia in Japan: a healthcare database analysis. Clin Neuropsychopharmacol Ther. 2011;2:1-8.

26. Ye W, Ascher-Svanum H, Tanji Y, Flynn JA, Takahashi M, Conley RR. Antipsychotic monotherapy among outpatients with schizophrenia treated with olanzapine or risperidone in Japan: a health care database analysis. Neuropsychiatr Dis Treat. 2012;8: 259-66.

27. Cheung S, Hamuro Y, Mahlich J, Nakahara T, Sruamsiri R, Tsukazawa S. Drug utilization of Japanese patients diagnosed with schizophrenia: an administrative database analysis. Clin Drug Investig. 2017;37:559-69.

28. Charlson ME, Pompei P, Ales KL, MacKenzie CR. A new method of classifying prognostic comorbidity in longitudinal studies: development and validation. J Chronic Dis. 1987;40:373-83.

29. Oya K, Kishi T, Iwata N. Efficacy and tolerability of aripiprazole once monthly for schizophrenia: a systematic review and meta-analysis of randomized controlled trials. Neuropsychiatr Dis Treat. 2015;11: 2299-307.

30. ABILIFY ${ }^{\circledR}$ [package insert]. Japanese. https://www. otsuka-elibrary.jp/pdf_viewer/?f=/file/1003/ abcbnotk.pdf. Accessed 18 Dec 2019.

31. Kamijima K, Higuchi T, Ishigooka J, et al. Aripiprazole augmentation to antidepressant therapy in Japanese patients with major depressive disorder: a randomized, double-blind, placebo-controlled study (ADMIRE study). J Affect Disord. 2013;151: 899-905.

32. Nelson JC, Papakostas GI. Atypical antipsychotic augmentation in major depressive disorder: a metaanalysis of placebo-controlled randomized trials. Am J Psychiatry. 2009;166:980-91.

33. Fujii Y, Iwata N, Takahashi K, Study group on relapse prevention of schizophrenia. Large-scale survey about psychiatrists' use, knowledge and attitudes to depot antipsychotics-a comparison between Japanese and German psychiatrist. Jpn J Clin Psychopharmacol. 2012;15:797-810 (Japanese).

34. Sugawara N, Kudo S, Ishioka M, Sato Y, Kubo K, Yasui-Furukori N. Attitudes toward long-acting injectable antipsychotics among patients with schizophrenia in Japan. Neuropsychiatr Dis Treat. 2019;15:205-11.

35. Malla A, Tibbo P, Chue $\mathrm{P}$, et al. Long-acting injectable antipsychotics: recommendations for clinicians. Can J Psychiatry Rev Can Psychiatr. 2013;58:30S-5S.

36. Taylor D, Paton C, Kapur S, editors. The Maudsley prescribing guidelines in psychiatry. 11th ed. London: Wiley-Blackwell; 2012.

37. Tiihonen J, Haukka J, Taylor M, Haddad PM, Patel MX, Korhonen P. A nationwide cohort study of oral and depot antipsychotics after first hospitalization for schizophrenia. Am J Psychiatry. 2011;168: 603-9.

38. Suzuki T. A further consideration on long-acting injectable versus oral antipsychotics in the treatment of schizophrenia: a narrative review and critical appraisal. Expert Opin Drug Deliv. 2016;13: 253-64. 